\title{
Research on the Data Synchronization of Cloud Stroke based on JSON
}

\author{
Hua Gu ${ }^{1}$, LeiHuang ${ }^{2}$, Jing Liu ${ }^{3}$ Qiuli Qin ${ }^{4}$ \\ Beijing Jiaotong University \\ No.3 Shang Yuan Cun Hai Dian District \\ Beijing, China 100044 \\ 1'guh@mail.bsti.ac.cn, ${ }^{2}$ lhuang@bjtu.edu.cn \\ ${ }^{3} 13120625 @ b j t u . e d u . c n^{4}$ qlqin@bjtu.edu.cn
}

\begin{abstract}
Cloud stroke is the cloud platform according to the medical association theory and combing cloud computing technology, build for the hospital systems which are involved in the medical association to sharing and exchange medical information. However, at this stage, there are some problems in the data exchange of the cloud platform: Firstly, every hospital in the medical association needs to configure a front machine and the front machine needs to connect with the cloud platform database directly. So there is a hidden risk for the cloud stroke. Secondly, the data synchronization vector is Excel file, the data reading efficiency is not very well, and at the same time, the front machine memory consumption is relatively very large. This paper, improve the data interactive process of cloud stroke, so that the front machine unneeded to connect with the cloud platform database directly. Then, design data specification and synchronization process based on JSON for cloud stoke information synchronization to compress the expenses, improve the speed and analysis the security of data synchronization.
\end{abstract}

Keywords: JSON • data synchronization.

\section{Introduction}

In order to deepen the reform of the medical and health system, establish and improve the basic medical and health system, accelerate the development of medical and health services, priority to meet the basic health needs of the masses, optimize the allocation of resources, increase efficiency and quality of information utilization. In the implementation of the national "Twelfth Five Year Plan", the concept of Medical Association is put forward. That is a general hospital unite regional secondary hospitals and community health service centers to form a medical associations. Residents choose the nearest association, firstly treated at community and referral step and step ${ }^{[1]}$.

The wide application and development of information technology in medical and health field, break the barriers between the inter agency and inter profession, provides medical resource sharing. In recent years, the "cloud computing" technology is developing rapidly. It promote the development of medical, health information and implementation of medical collaboration network.

The theme of this dissertation is derived from the topic "Construction and demonstration application of the joint body model of major disease prevention and control in the medical reform in Beijing City" (No. Z13110000561317). The topic choice stroke as the breakthrough point, cooperative prevention and control of stroke. Acupuncture on stroke medical association and combined cloud computing technology, we build a cloud stroke whose function is to share and exchange medical information for the hospitals in the medical association. However, at this stage, there are still some problems in the cloud 
stoke: it is limited to expand the function of data exchange, data exchange has security hidden trouble and data exchange resource consumption is relatively large.

In this paper, through research on the cloud platform for data synchronization status and the data synchronization base on JSON, we propose a data synchronization method based on JSON for the cloud stoke, so that we can improve the data synchronization of cloud stoke.

\section{The Cloud Stoke Present Status of Data Synchronization}

The medical treatment body of cloud stroke is combined by Tiantan hospital, Fangzhuang community health service center and emergency center. The date of cloud stroke mainly comes from the three hospitals. Treatment is divided into multiple stages: first level prevention, first aid management, hospital management, rehabilitation management, two level prevention. In order to complete the data of patients with stroke in each stage, the cloud stroke needs to collected, transfer and storage the data saved in the different hospital information system.

\subsection{Data Synchronization Process}

As there are limitations between hospital network and public network, and in view of the security requirements of medical data. We need to deploy a front machine in every hospital to collect data. At the present stage the front machine need to realize the function of data collection, transfer, and temporary store and then push the temporary store data to the cloud stroke. The cloud stroke will save the data in the database which was built in the platform.

The process of cloud platform data synchronization is as below:

$$
\text { Hospital }
$$

Cloud platform

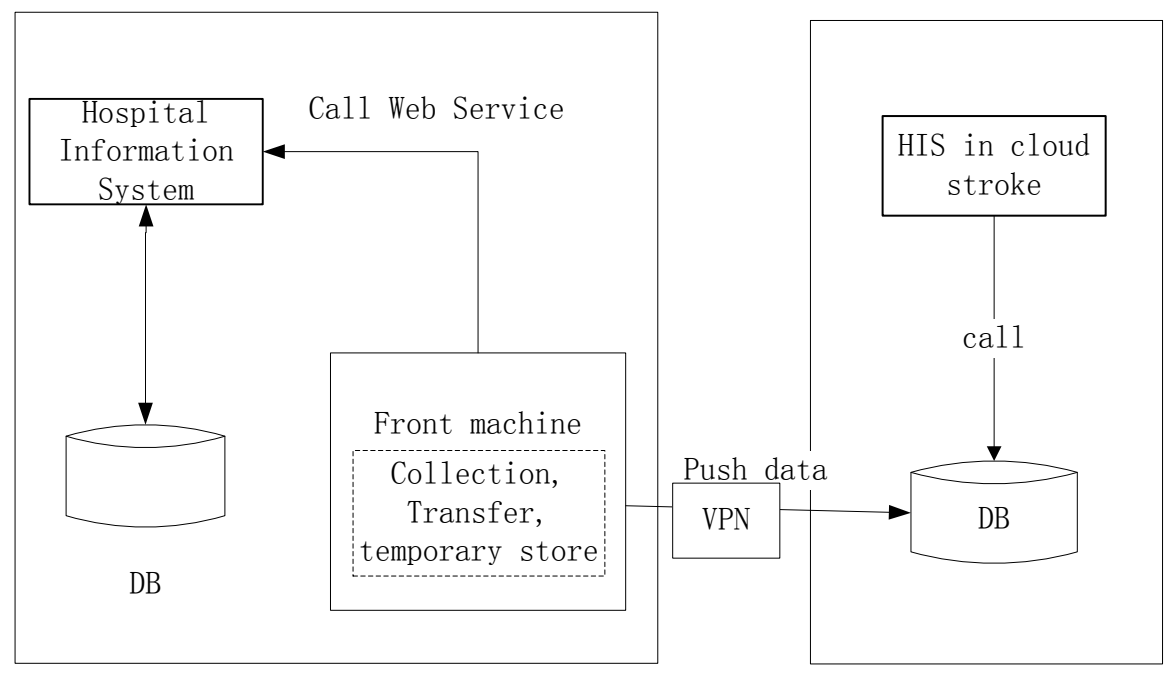

Figure 2-1. Data synchronization process ${ }^{[2]}$

\section{(1) Data acquisition}

Data acquisition services deployed on the preprocessor deployed within front machine to call the Web Service interface services provided by each hospital, so that the front machine can get the data obtained from the database of the business application system of each hospital.

\section{Data push}


The front machine package the collected data and then push the packaging data to the cloud stroke through a custom message communication protocol.

$$
\text { Data conversion }
$$

After the data was pushed into the cloud stroke, the Information sharing system's data conversion services will transform and enrich the data within standard content types.

$$
\text { Data storage }
$$

Storage the data into the database of cloud stroke.

\subsection{Data Synchronization Implementation Mechanism}

In the existing management information system of hospital, Data is stored in a structured and semi structured format. This project chooses Excel as the middle ware, at the same time, build the data exchange interface to realize the data exchange between the hospitals in medical association and cloud stroke. The data synchronization implementation mechanism is as follows:

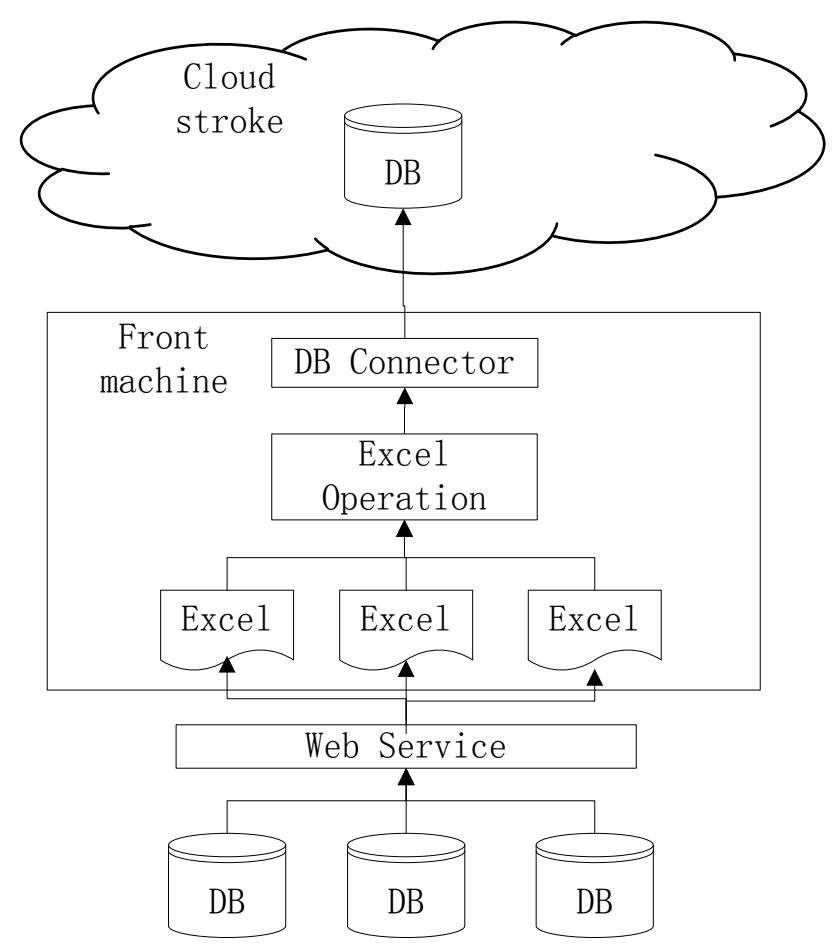

Figure 2-2. The Data Synchronization Implementation Mechanism

The hospital information systems in medical association provide web service interface for the front machine. The front machines call the interface to store the data which is needs to be synchronized in the Excel file. Excel operator reads the contents of the Excel file and transform and enrich the data within standard content types, then directly connected to the database for storage.

With the research on data synchronization process and data synchronization implementation mechanism of cloud stroke, we found that there are shortcomings of data synchronization.

(1) There is safety hidden danger as the front machine connect the database of cloud stroke directly. 
(2) Although it is easily to be understood the data by pushing the data using Excel file, it consumes large memory of reading and writing content into the Excel file. At the same time the front machine load too much functionality, the front machine is so overloaded.

\section{The Structure and Advantages of JSON}

JSON (JavaScript Object Notation) is a lightweight text-based data interchange format that is easy for both humans and computers to digest and consume ${ }^{[3]}$.

JSON has six types of data ${ }^{[4]}$ :

Four basic types: String, numeric, Boolean, Null value.

Two structure types: object, the key's type is String and the value can be any type of JSON; Array, several sequence of JSON data.

Among the types of JSON the object is the most flexible type in JSON applications and it structure is as follows:

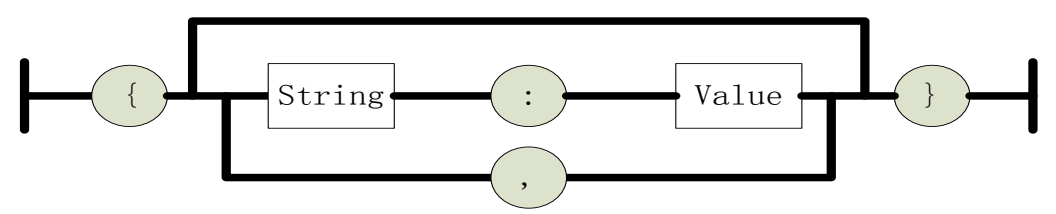

Fig. 3-1 JSON Object Structure

Object is a "key / value" pair set and is an unordered collection. An object is started with "\{" and end with "\}". Each "key" is followed by ":" and each object is separated by ",".

In the application of JSON, Arfon Smith (2014) use JSON to realize the data encapsulation and delivery between API, because it has the advantages of Simple and without ambiguity ${ }^{[5]}$. Xuhui Li,Mengchi Liu (2014) use JPQ which was created based on the Grammatical patterns and data structures of JSON to realize the information exchange between different files ${ }^{[6]}$.

In the specification of JSON, Alexander Keith (2008) did several corresponding research on JSON specification ${ }^{[7]}$. Galiegue and K. Zyp (2013) defined the data structure based on JSON by making the JSON Schema documents and descripting the application scenarios of JSON, provided the application requirements and interaction of JSON data. So that we can make validation specification, document description, hyperlink navigation and interactive control aim at JSON data ${ }^{[8]}$. Bray $\mathrm{T}$ (2014), deleted the inconsistent specification in JSON which is different with other transmission structures, repaired specification error and provides experience based interoperability guidance ${ }^{[9]}$.

In the secure transmission of JSON, Y Zhishu, L Yanyan, M Jun (2008) proposed a JSON security architecture model based on SSO to implement the authentication of single sign ${ }^{[10]}$. El-Aziz A A Kannan, A Abd (2014) in the study described how to shift the XML encryption to JSON encryption ${ }^{[11]}$.

With the research on JSON and the data exchange by JSON, we found that JSON is Simple, high compressibility, high availability and wide range support.

\section{Improve the Method of Cloud Stroke's Data Synchronization based on JSON}

The original synchronization method uses the excel document to synchronous data timing. In this paper, we use JSON technology to transform the existing technology of data synchronization. Finally change the information into JSON format synchronization. At the same time, create an external access mechanism for cloud platforms, so that 
doctors and patients with different permissions to access the cloud platform data. To facilitate the view of medical information. Prior to this need to improve the cloud platform and the hospital data synchronization architecture, in order to achieve the desired results.

\subsection{The Improvement of Data Synchronization Architecture}

In order to avoid the front machine connected the database in cloud stroke directly. This paper improve the synchronization architecture as follows:

Hospital

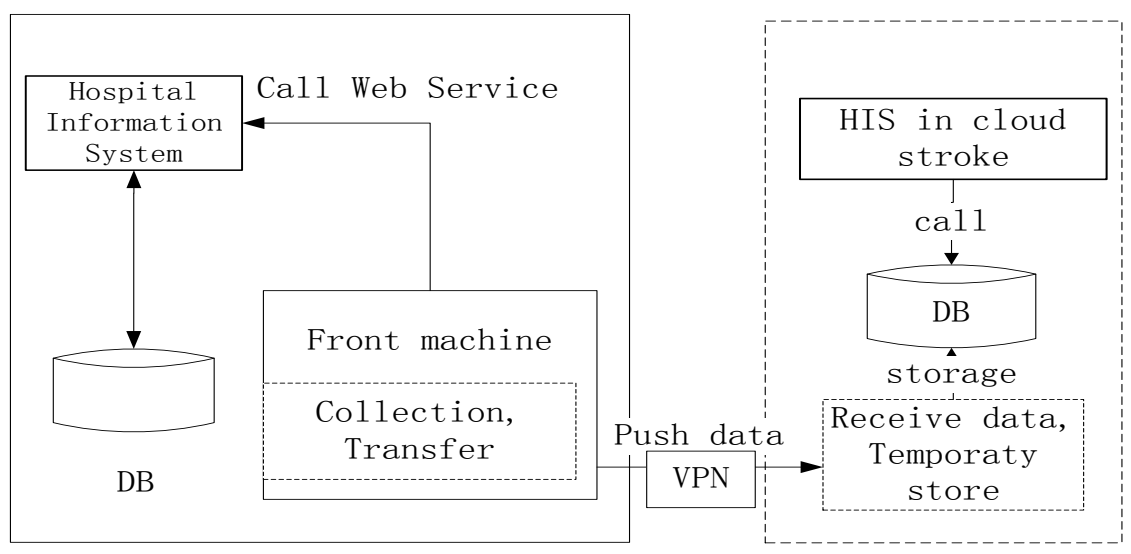

Figure 4-1. The Architecture of Data Synchronization

After improvement, the front machine only responsible for data acquisition and conversion. Converted data in the form of post parameters through the VPN sent to the cloud platform web services, to synchronize by the cloud platform. After receiving the sync request, cloud stroke analyzes the parameters and then storage the data into the data base in cloud stoke, completes the data synchronization.

\subsection{The Improvement of Data Synchronization Method based on JSON}

As the original synchronization method uses the excel document to Synchronous data timing. The front machine need consuming large resources. This paper uses JSON as the structure of parameters to complete the data synchronization. The specific structure design and implementation mechanism are as follows.

(1) The design of medical information transmission structure based on JSON

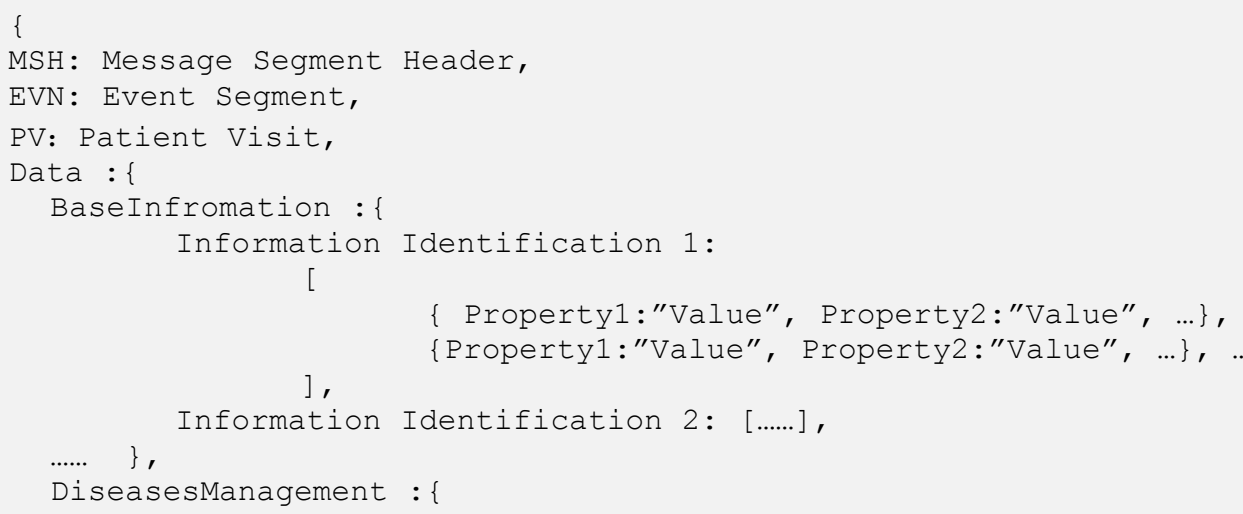




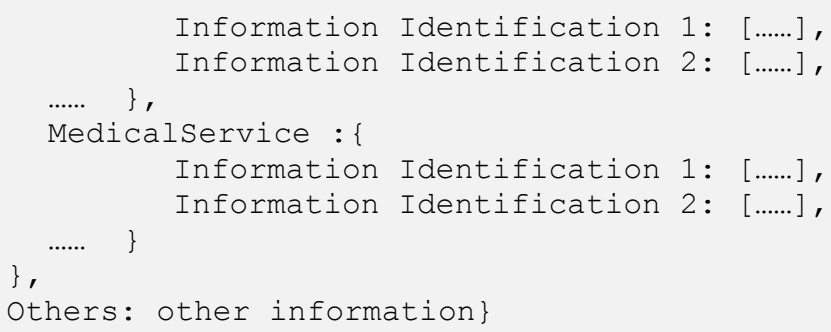

The JSON data includes MSH, EVN, Data and Others.

MSH, Message Segment Header, storages the base information of data.

EVN, Event Segment, decides how to deal with transactions. Different hospital information system has different design about "Hospitalized patient", "Outpatient", "Emergency room", "Recurrent patient", and so on. And there will be different treatments for these patients. So event segment does not be designed for any patient visit. That means how to handle the event, the platform needs to check the event and the patient visit.

PV, Patient Visit.

Data, the detail information of medical. It include base information, diseases management and medical service. According to the event and patient visit, there will be different information included in the data.

Others, other information needed.

(2) The implementation mechanism based on JSON

The detail implementation mechanism based on JSON is as follows:

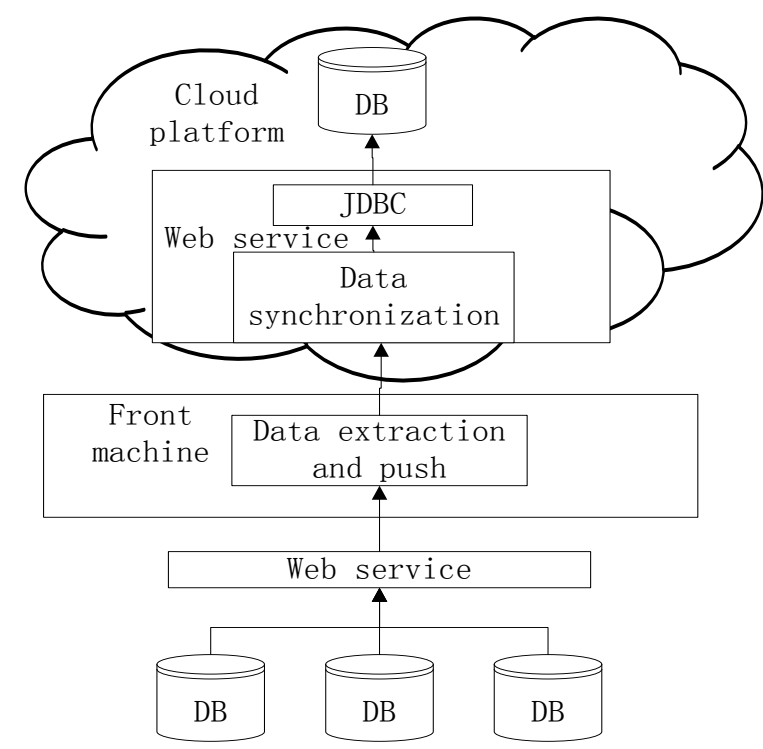

Fiure 4-2 The Implementation Mechanism of Data Synchronization

After implement, the interface of data extraction in different hospital's web service does not need be changed. The front machine call interface, the data extracted by the Operation Data module for processing. The extracted data is constructed as the information of the transmission structure shown in the last section, which is based on the JSON transmission structure. The data is put into the post request of the cloud platform Service web in the form of the parameters. After receiving the request, the data synchronization module in the cloud platform is used to parse the parameters of the 
request and data mapping with cloud platform. Then storage the data into the database of cloud stroke.

\section{The Realization of Cloud Stroke's Data Synchronization based on JSON}

This section will build the medical information and data access request information for cloud stroke by using the transmission structure and access information structure designed in this paper. At the same time, compared with the original Excel document transmission mode, to see whether the improvement of the efficiency of the data synchronization is improved.

\subsection{Construction of JSON Message}

Synchronous medical information with the health file table as an example, to build the JSON message format as follows:

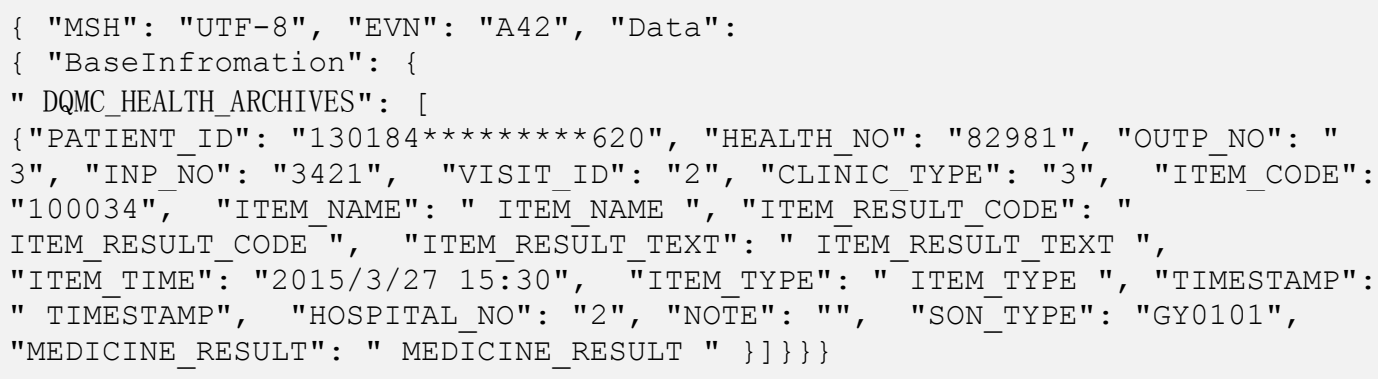

Then store the corresponding field according to the key word in the message.

\subsection{Implementation of Data Synchronization Interface}

Taking the health file information as an example, construct the health archives collection information to the JSON information, and implement the interface of changing the JSON information to the local information.

The construction program of JSON information:

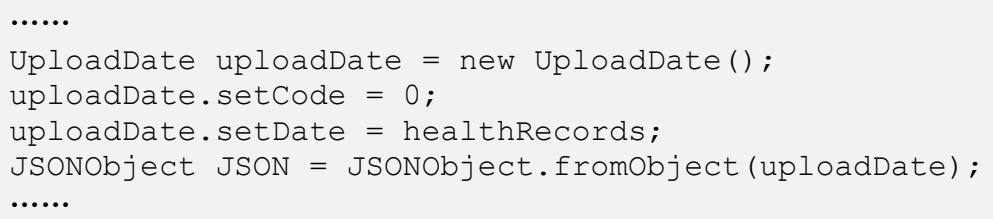

In the interface, the program uses createHealth RecordInfor method to construct the JSON information. Then JSON message will be transmitted to the cloud platform in the form of string.

The analysis program of JSON information

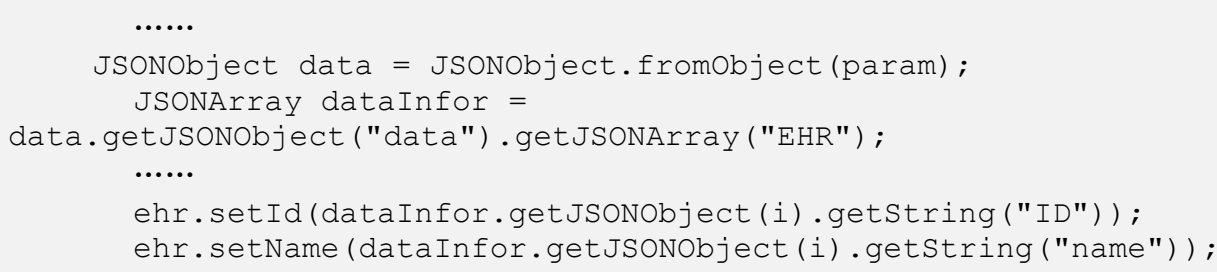




\subsection{The Comparison of Synchronization Efficiency}

In order to ensure the reference of the test results, the Excel data analysis and JSON data analysis test cases are set up in the same environment. Test data quantity are 500, $1000,2000,3000,4000$. At the same time, in order to avoid the error of the single test, for different test data, we have conducted 10 tests. The analysis time and memory consumption are monitored.

The comparison of Synchronous data parsing time and synchronization is the memory consumption are as follows:

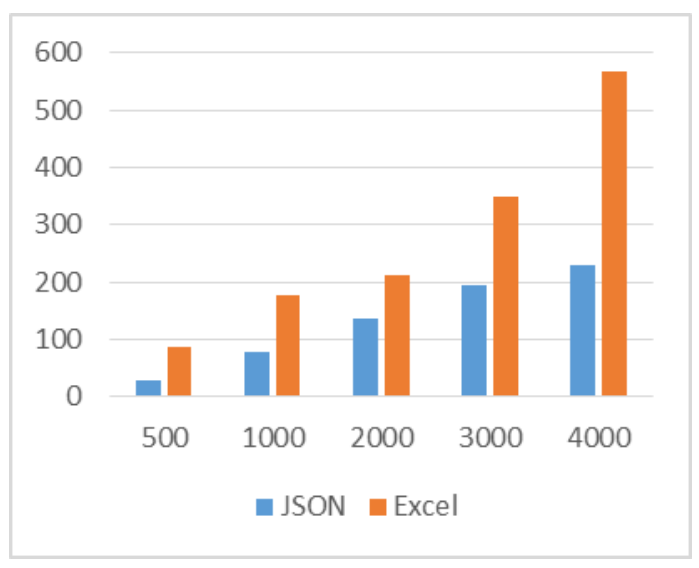

Figure 5-1 The Histogram of

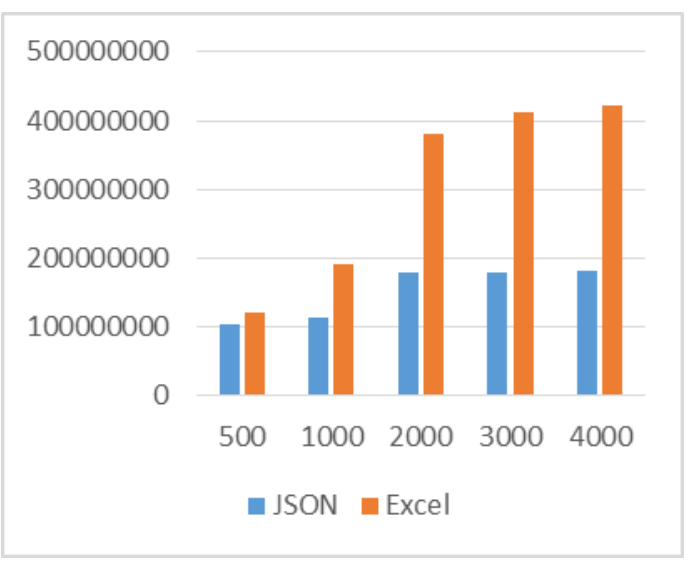

Figure 5-2 The Histogram of Parsing Memory Consumption

Blue represents the analytical data's format is JSON, the orange represents the analytic object is the Excel format. The horizontal coordinates represent the test data quantity and the vertical coordinates represent the test results. We can draw the following results from the two histograms. The average time parsing JSON format data is significantly less than parsing Excel format data. The parsing time increases with the increase of the amount of data that needs to be resolved, but the parsing time increases much more fast by using Excel to format data. With the increase of the amount of data the average time gap between the two is also increased. Using JSON data transfer data analysis time is significantly shortened visibly and the memory consumption is also greatly reduced.

\section{Conclusion}

In this paper, through the analysis of cloud stroke's data synchronization status, found deficiencies in the data synchronization. Firstly, improve the cloud stoke data synchronization architecture, avoid the front machine connects the cloud stoke database directly, enhanced platform data's security. Then, through the research of JSON data format, for the lack of cloud stroke's data synchronization, reconstruct the data synchronization information based on JSON and then applied to the cloud stroke's data synchronization. Combined with the specific medical information using the analytical method of the original data and the medical information based on JSON to build the analytical method of time and memory consumption. The result is that based on JSON 
medical information synchronization not only shorten the time synchronization, parsing the memory consumption is also greatly reduced.

\section{Acknowledgements}

The authors would like to express their great thanks to the support from the Fundamental Research Funds for education, number 2013JBM038, and the Logistics Technology and Management Lab.

\section{References}

[1] Huaqin Chen, Application of SWOT in Medical Associations of Hospitals, Modern Hospital Management, vol. No. Dec 6 (2013)

[2] Bei Xi, The Research of the Stroke Medical Association Information Sharing Platform Based on the Cloud Computing, Beijing Jiaotong University (2015)

[3] Hailong Xu, Research on data exchange technology and its application in Web. Huazhong University of Science and Technology, DOI:10.7666/d.d190872 (2011)

[4] Siwei Xing. Research and implementation of information interaction system based on JSON. Anhui University (2013)

[5] Arfon Smith. JSON-LD for software discovery, reuse, and credit, (2014). http://www.arfon.org/json-ldfor-software-discovery-reuse-and-credit Accessed: 2014-07-10.

[6] Xuhui Li, Mengchi Liu, Xiaoying Wu, Shanfeng Zhu:Design Issues of JPQ: a Pattern-based Query Language for Document Databases. (2014)

[7] Keith Alexander.RDF/JSON: A Specification for serialising RDF in JSON. Proceedings of the 4th Workshop on Scripting for the Semantic Web CEUR Workshop Proceedings . (2008).

[8] Galiegue and K. Zyp. JSON Schema: core definitions and terminology. draft-zyp-json-schema-04 (work in progress), (2013). URL https://tools.ietf.org/html/draft-zyp-json-schema-04.

[9] Bray T. The JavaScript Object Notation (JSON) Data Interchange Format[J]. (2014).

[10] Jun Y, Zhishu L, Yanyan M. Json based decentralized sso security architecture in ecommerce[C]//Electronic Commerce and Security, 2008 International Symposium on. IEEE, (2008): 471-475.

[11] Abd El-Aziz A A, Kannan A. JSON encryption[C]//Computer Communication and Informatics (ICCCI), (2014) International Conference on. IEEE, 2014: 1-6. 
International Journal of Grid and Distributed Computing

Vol. 9, No. 7 (2016) 